\title{
NUEVOS RETOS EN LA EDUACIÓN. EL IMPULSO DE METOdOLOGíAS ALTERNATIVAS APRENDIZAJE-SERVICIO Y MENTORÍA
}

\author{
Mirian Hervás Torres \\ miriamhervas@ugr.es \\ M.Tamara Polo Sánchez \\ tpolo@ugr.es \\ Carolina Fernández Jiménez \\ María Fernández Cabezas \\ Susana Tallón Rosales
}

Fecha de Recepción: 9 Marzo 2018

Fecha de Admisión: 10 Abril 2018

\section{RESUMEN}

La Educación Superior se enfrenta al desafío de mejorar la productividad y competitividad del alumnado, además de revisar cuál es su función social. Para la mejorar la calidad de la educación y ser más competentes en la sociedad lleva asociado potenciar las metodologías docentes alternativas que permiten conectar el currículum con la realidad, caso del Aprendizaje-Servicio y la mentoría. En esta línea, el objetivo de este trabajo fue explorar la eficacia del Programa Huelva Educa para mejorar la motivación, estrategias de aprendizaje, y habilidades sociales de los mentores participantes y rendimiento escolar y clima escolar de los "mentees". La muestra estuvo compuesta por 79 estudiantes de la Universidad de Huelva, y 69 alumnos de educación obligatoria seleccionados a través de un muestreo no probabilístico de conveniencia. Los resultados muestran que el alumnado universitario, tras su participación, mejora en comportamientos relacionados con estrategias de aprendizaje (p.e., tiempo y ambiente de estudio, autorregulación metacognitiva o regulación del esfuerzo), y el alumnado de educación obligatoria ha incrementado la cantidad y calidad de sus interacciones, así como tendencias de mejora en el rendimiento escolar. Estos resultados, sugieren la necesidad de implantar de este tipo de programas basados en metodologías alternativas dentro del proceso de cambio que está sufriendo la Educación Superior.

Palabras clave: aprendizaje-servicio; mentoría; educación superior; competencias

\section{ABSTRACT}

New challenges in education. The impulse of Service-Learning and Mentoring alternatives methodologies.

Higher Education is facing the huge challenge of improving their productivity and competitive- 
ness of the students, in addition to check what is its social function. Improving the quality of education and be more competent in the society is associated to promote alternative educational methodologies that allow connecting the curriculum with the reality, case of Service-Learning and mentoring. In this sense, the aim of this work was to explore the effectiveness of the Huelva Educa Program to improve the motivation, strategies of learning and social skills of mentors and school performance and school climate of "mentees". The sample was composed of 79 students from the University of Huelva, and 69 compulsory students selected through a sampling non-probability convenience. The outcome shows that university students who participated, they have significantly improved behaviors related to learning strategies (e.g., time and environment study, metacognitive self-regulation or regulation of the effort), and de compulsory students has increased the quantity and quality of their interactions, as well as improvement in school performance. These outcomes suggest the necessity of the implementation of such programs based on alternative methodologies within the process of change that is suffering Higher Education.

Keywords: service-learning; mentoring; higher education; competencies

\section{INTRODUCCIÓN}

La situación del sistema educativo español, presenta una realidad dura. Los datos sobre Educación Primaria (EP), indican que un 4,5\% de alumnos repiten en $2^{\circ}$ curso, el 3,7\% lo hace en $4^{0}$ curso y el 4,0\% en 6은 curso (Ministerio de Educación, Cultura y Deporte, 2017), además del bajo nivel en competencias clave registradas (p.e.: comprensión lectora, matemáticas y ciencias) (Instituto Nacional de Evaluación Educativa, 2015). En Educación Secundaria Obligatoria (ESO), Ios porcentajes varían dependiendo del curso, encontrando que el $12,3 \%$ del alumnado repite en $1^{\circ}$ curso, el $10,7 \%$ en $2^{\circ}$ curso, un $11,0 \%$ en $3^{\circ}$ curso y en $4^{\circ}$ un $9,5 \%$, situando la tasa de abandono en el 19,0\% (Ministerio de Educación, Cultura y Deporte, 2017). A esto se unen las deficiencias en competencias clave, como reflejan los resultados globales en competencias en matemáticas (484), comprensión lectora (488), ciencias (496) y resolución de problemas (477) (Instituto Nacional de Evaluación Educativa, 2015).

El escenario en el ámbito universitario tampoco mejora (p.e., tasa de abandono del estudio en primer año de la cohorte que comenzó sus estudios de grado en el curso 2012-2013 del 20,5\%, tasa de cambio del estudio del $7,5 \%$, tasa de idoneidad y graduación en grados de 4 años de duración es del 33,2\% y 49,6\%, respectivamente) (Ministerio de Educación, Cultura y Deporte, 2017). Asimismo, la calidad de la formación, la cual afecta al rendimiento académico diferido, es decir, el nivel de ocupación que alcanzan los egresados también está comprometido (p.e., el 55,5\% de los egresados 4 años después ocupan un puesto acorde a su titulación; opiniones negativas de los titulados respecto a las posibilidades que su formación ofrece para encontrar empleo y de los empresarios sobre la formación de los titulados; etc.) (Consejo Económico y Social de España, 2017; Freire, Teijeiro, y País, 2013; Ministerio de Educación, Cultura y Deporte, 2017). Esta realizada marca la necesidad de establecer medidas específicas de apoyo que reduzcan estas cifras, y buscar procedimientos que permitan aumentar las competencias, prevenir el fracaso y abandono, etc., del alumnado como es el caso del Programa Huelva Educa (PHE), el cual está basado, por un lado, en la metodología alternativa Aprendizaje-Servicio (A-S) (Westover, 2012; Wilczenski y Coomey, 2007), entendida como una metodología donde los participantes desarrollan habilidades, destrezas, competencias, etc., realizando un servicio a su comunidad. Y, por otro lado, el aprendizaje entre iguales, en su versión de mentoría (Sandford, Copps, y Yeowart, 2007), donde una persona con experiencia ayuda a otra más joven en aspectos esenciales de su vida.

Entre los beneficios de estas metodologías se destacan: (a) aumento del compromiso del alumnado con el aprendizaje (Maloney y Griffith, 2013; McIntyre y Sellnow, 2014), (b) mejora en la adqui- 
sición de los contenidos (Jackevicius, Le, Nazer, Hess, Wang, y Law, 2014; McIntyre y Sellnow, 2014; Weiler, Zarich, Haddock, Krafchick, y Zimmerman, 2014;), (c) fomento de aspectos relacionados con el desarrollo personal y social del alumnado (p.e., responsabilidad, autoestima, etc.) (Hervás, Fernández, Arco, y Miñaca, 2017; Jackevicius et al., 2014; Mclntyre y Sellnow, 2014; Weiler et al., 2014), (d) estímulo de la reflexión y el pensamiento crítico entre el alumnado (Stanton, 2014; Vaknin y Bresciani, 2013), (e) incremento del sentido de civismo (Jackevicius et al., 2014; Puig, Batlle, Bosch, y Palos, 2007; Weiler et al., 2014), (f) incremento de la motivación y satisfacción del profesorado (p.e., mejora el ambiente académico y contenido educativo, etc.) (Tapia, 2006; Wilczenski y Coomey, 2007), (g) mayor conexión de la enseñanza del aula con las necesidades del entorno (McIntyre y Sellnow, 2014), y (h) refuerzo de los lazos sociales y vínculos entre diferentes generaciones pertenecientes a una comunidad (Maloney, Myers, y Bazyk, 2014; McIntyre y Sellnow, 2014).

Por ello, una intervención basada en ambas metodologías, adquiere un papel protagonista, ya que se dirige a la integración y capacitación en competencias, mejora de la formación, rendimiento académico, y desarrollo personal, aportando aquellos elementos que sean óptimos y adecuados en la relación y percepción del alumnado hacia la escuela, ofreciendo las herramientas necesarias con las que disminuir el fracaso y abandono escolar, y en consecuencia su separación del sistema educativo.

En definitiva, el objetivo general fue explorar los efectos de la participación en el PHE, para mejorar la motivación, estrategias de aprendizaje, y habilidades sociales de los mentores y rendimiento escolar y clima escolar de los "mentees".

\section{MÉTODO \\ Participantes}

En este trabajo participaron 148 sujetos distribuidos en dos submuestras. Por un lado, 79 mentores de diferentes titulaciones pertenecientes a la Universidad de Huelva (UHU), con una media de edad de 21,22 años (rango de entre 18 y 34 años), donde la distribución por sexo fue de 67 mujeres $(84,81 \%)$ y 12 hombres $(15,19 \%)$. Y, por otro lado, 69 alumnos de EP y ESO, con una media de edad de 12,26 años (rango de edad entre los 10 y 15 años), donde su distribución por sexo fue de 30 mujeres $(43,47 \%)$ y 39 hombres $(56,53 \%)$.

El procedimiento de selección de las muestras se basó en un muestreo no probabilístico, denominado muestras de conveniencia (Martínez, 2010): (a) selección de la población a la que se tenía acceso (unidad primaria: Universidad españolas y centros educativos españoles), (b) selección de la población diana a la que se pediría participar en la investigación (muestra invitada) (unidad secundaria: titulaciones impartidas en las Universidad españolas elegidas en la etapa anterior y concreción de los ciclos educativos de EP y ESO impartidos en los centros elegidos en la anterior etapa), (c) fragmento de las muestras invitadas que aceptaron participar, muestras participantes, (unidad terciaria: alumnado de titulaciones seleccionadas en la etapa anterior y alumnado del tercer ciclo de EP y primer ciclo de ESO de los centros educativos seleccionados en la etapa anterior que se registraran voluntariamente), y (d) fracción de las muestras participantes seleccionadas para la investigación, muestra final, (unidad cuaternaria: alumnado universitario y alumnado de EP y ESO registrado en el PHE en la etapa 3 que voluntariamente se inscribió o fue inscrito en el programa).

\section{Instrumentos}

Acuerdo de participación mentores. Documento elaborado ad hoc desarrollado a partir de otros materiales similares (Fernández, 2007; Hervás et al, 2017), con el propósito de establecer los derechos y deberes del alumnado universitario participante. 
Protocolo de recogida de información mentores. Inventario desarrollado ad hoc cuyo objetivo fue en recoger información demográfica, académica, profesional o familiar, de capacitación e interés de los alumnos universitario interesados en participar. Se adaptó de otras experiencias (Fernández, 2007; Hervás et al., 2017).

Protocolo para apoyo externo "mentee". Inventario elaborado ad hoc, adaptado de Fernández (2007), donde su propósito fue recoger información demográfica y escolar, y de interés del alumnado de educación obligatoria.

Protocolo de seguimiento. Instrumento donde se identificó la valoración-percepción de las sesiones realizadas. Fue adaptado de otros estudios (Fernández, 2007) y se usó para identificar las posibles desviaciones que se originaran en el desarrollo de las sesiones de mentoría.

Cuaderno de mentores. Material que presentaba los objetivos, tareas realizadas, materiales empleados, dificultades encontradas, tareas pendientes para la próxima sesión y evaluación de la sesión y observaciones. Fue adaptado y desarrollado a partir de otros materiales y trabajos de investigación (Hervás et al., 2017) y se empleó durante las sesiones de mentoría y el plan de seguimiento del programa.

Pruebas estandarizadas. Diferentes pruebas estandarizas con las que comprobar la eficacia del PHE.

\section{Procedimiento}

El diseño metodológico adoptado fue de carácter cuasi-experimental (Ato, 2010), el cual se desarrolló en cuatro fases: análisis de necesidades, diseño y planificación, aplicación y evaluación del programa (Arco y Fernández, 2007).

Seleccionadas la muestra, se inició el plan de intervención del programa, el cual constaba de dos actividades, el curso de formación de mentores y las sesiones de mentoría (Hervás et al., 2017). La primera actividad, el curso de formación, se dirigió a la formación de los mentores en contenidos que deberían estar presentes en este tipo de programas, con la finalidad de mejorar el nivel de competencias en actividades de mentoría (Mentor/National Mentoring Partnership, 2005). Finalizado el curso se formación de mentores, se seleccionó la muestra de mentores y "mentees", los cuales fueron inscritos por sus docentes-tutores, con el previo consentimiento de sus familias, procediendo al emparejamiento entre mentores y "mentees", considerando la disponibilidad horaria y la equivalencia en experiencia de vida e intereses (Mentor/National Mentoring Partnership, 2005).

Conformadas las parejas, se iniciación las sesiones de mentoría (segunda actividad del plan de intervención), desarrollándose en horario extraescolar durante el curso escolar, en formato individual (cada mentor con su respectivo alumno de educación obligatoria) y grupal (varios mentores con sus respectivos alumnos de educación obligatoria en actividades comunes). La frecuencia de las sesiones de mentoría era semanal y con una duración aproximada de 90 minutos, realizándose en los horarios fijados previamente, y en los lugares habilitados por los responsables del programa (aulas de los centros educativos de referencia 0 aulas proporcionadas por la UHU). Asimismo, las actividades llevadas a cabo (Cuaderno de mentores) durante las sesiones de mentoría se adecuaron a sus necesidades, la cuales fueron identificadas a partir de la información aportada por los docentes-tutores (Protocolo para apoyo externo "mentee"), y la información recogida por los mentores durante la primera sesión de mentoría, en los términos que recomienda la literatura especializada (Boland, 2010; Miñaca y Hervás, 2013), dirigiéndose hacia: (a) refuerzo escolar en áreas instrumentales (p.e., intervención en dificultades de aprendizaje, etc.), (b) actividades de orientación personal (p.e., habilidades sociales, etc.), (c) actividades de orientación vocacional-profesional (p.e., itinerarios formativos, etc.), y (d) actividades de gestión del ocio y tiempo libre (p.e., resolución de conflictos, etc.). 
Se realizaron 955 sesiones de mentoría ( $\bar{X}=13,84 ; \sigma X=2,51$; rango entre 9 y 18 sesiones), de las cuales 878 fueron de carácter individual ( $\bar{x}=12,72$; $\sigma_{X}=2,90$; rango entre 4 y 18 sesiones), y 77 de carácter grupal ( $\bar{x}=1,11$; $\sigma \mathrm{X}=1,66$; rango entre 0 y 10 sesiones). No obstante, las diferencias en el número de sesiones de mentoría realizadas se deben a los diferentes momentos en los que el alumnado de educación obligatoria fue inscrito, así como a las sesiones de mentoría que fueron canceladas $(N=184)$ por diferentes motivos, tanto individuales $(N=132 ;=1,91 ; \sigma X=, 78$; rango entre 1 y 4 sesiones) como grupales ( $N=52 ; \bar{x}=, 75 ; \sigma X=, 73$; rango entre 0 y 3 sesiones).

Paralelamente al desarrollo de las sesiones de mentoría, con la finalidad de identificar posibles dificultades respecto al desarrollo de las sesiones, se llevó a cabo el plan de seguimiento (Arco y Fernández, 2007): (a) 25 sesiones grupales con los docentes-tutores del alumnado de educación obligatoria con una frecuencia de 1 sesión mensual de 1 hora para conocer la evolución del alumnado en términos académicos, (b) 25 sesiones grupales con las familias del alumnado de educación obligatoria, de 1 hora de duración aproximadamente y con una frecuencia de 1 sesión mensual, para conocer la evolución del alumnado en el ambiente familiar, y (c) 8 sesiones con los mentores, de carácter grupal y de 2 horas de duración, que se efectuaron una vez realizada la segunda (sesión 1 de seguimiento), la séptima (sesión 2 de seguimiento) y la duodécima sesiones de mentoría (sesión 3 de seguimiento), así como al finalizar las sesiones de mentoría (sesión 4 de seguimiento), donde se valoraron las diversas actuaciones implementadas en las sesiones de mentoría y se recogieron sus percepciones respecto a la eficacia de las mismas facilitándoles las recomendaciones oportunas para el desarrollo de las siguientes sesiones de mentoría.

Por último, mediante el plan de evaluación de resultados se tomaron medidas de las variables dependientes una vez finalizó la aplicación del programa para conocer el impacto del mismo (Arco y Fernández, 2007).

Los datos fueron analizados a través del paquete estadístico "Statistical Package for the Social Sciences" (SPSS) para Windows, versión 22.0, realizándose los siguientes análisis: (a) prueba Kolmogorov-Smirnov, (b) prueba de los rangos con signo de Wilcoxon, (c) prueba $t$ para muestras relacionadas, (d) estadístico de McNemar, y (e) valor $d$ de Cohen.

\section{RESULTADOS}

\section{Resultados mentores}

Los resultados registrados sobre aserción y habilidades sociales en la fase pretest y postest revelan un aumento de la media de puntuación directa obtenida en 4 de las 6 subescalas de la Escala de Habilidades Sociales [EHS] (Gismero, 2000), así como en la puntuación directa global, apreciándose diferencias estadísticamente significativas en la subescala iniciar interacciones positivas con el sexo opuesto $(z=-2,82 ; p<0,01)$ y en la puntuación directa global $(z=-2,28 ; p<0,05)$, y al mismo tiempo, se ha registrado un tamaño del efecto entre ambas medidas pretest y postest de las subescalas que oscila entre los 0,02 y 0,17 puntos.

Los resultados sobre motivación y estrategias de aprendizaje, registran un aumento de la media de puntuación directa en 14 de las 15 subescalas del Motivated Strategies for Learning Questionnaire [MSLQ] (Pintrich, Smith, García, y McKeachie, 1991) (exceptuando la subescala orientación intrínseca), apreciándose diferencias estadísticamente significativas en las subescalas de orientación extrínseca $(z=-2,36 ; p<0,05)$, ansiedad $(z=-3,57 ; p<0,001)$, PC $(z=-3,59 ; p<$ $0,001)$, tiempo y ambiente de estudio $(z=-2,05 ; p<0,05)$, autorregulación metacognitiva $(z=-3,53$; $p<0,001)$, regulación del esfuerzo $(z=-4,23 ; p<0,001)$ y solicitud de ayuda $(z=-2,98 ; p<0,01)$ y un tamaño del efecto que oscila entre 0,06 y 0,49 puntos. 


\section{Resultados "mentees"}

Los resultados sobre rendimiento escolar revelan una evolución positiva en las calificaciones obtenidas, con un incremento de asignaturas aptas entre el alumnado de EP (196 asignaturas aptas en el primer trimestre, frente a 209 en el tercer trimestre). Además, de un mantenimiento estable en las calificaciones obtenidas en el alumnado de ESO (296 asignaturas aptas en el primer trimestre frente a 281 en el tercer trimestre). Asimismo, en el segundo trimestre, para el alumnado de EP, se aprecian tendencias de mejora en el área de Lengua castellana y literatura, y para el alumnado de ESO en el área de Tecnología y en el tercer trimestre se aprecian diferencias estadísticamente significativas en el área curricular de Conocimiento del medio natural, social y cultural en el alumnado de EP y tendencias de mejora en las áreas curriculares instrumentales (Lengua castellana y literatura, y matemáticas).

Los resultados sobre las características socio-ambientales y relaciones personales en el aula revelan que la media de puntuación directa conseguida en 8 de las subescalas de la Escala de Clima Social [CES] (Moos, Moos, y Trickett, 1995), se ha incrementado en la fase postest, si bien en la subescala innovación se aprecia una mayor puntuación en la fase pretest $\left(\bar{X}=5,80 ; \sigma_{X}=1,41\right)$ frente a la fase postest $\left(\bar{X}=5,62 ; \sigma_{X}=1,71\right)$. Estos resultados no arrojan diferencias estadísticamente significativas entre las puntuaciones medias obtenidas en ninguna de las subescalas, mientras que los resultados del valor $d$ de Cohen oscilan entre 0,00 y 0,32 puntos.

\section{DISCUSIÓN}

Este estudio se dirigió a conocer la eficacia del PHE, un programa basado en la metodología alternativa A-S y mentoría, para mejorar la motivación, estrategias de aprendizaje, habilidades sociales, rendimiento y clima escolar. De acuerdo con los resultados, se observan mejoras, pero sin diferencias estadísticamente significativas, aunque como recomiendan otros autores (Ledesma, Macbeth, y Cortada, 2008) los resultados de este tipo de investigaciones deben interpretarse también en función del tamaño del efecto, de cara a identificar su significatividad práctica.

En este punto, los resultados indican una mejora en las variables dependientes, pero con la conveniencia de realizar un análisis más profundo para comprobar si las mejoras en motivación, estrategias de aprendizaje y habilidades sociales se reflejan en el progreso académico y personal de los mentores, como indica la literatura especializada sobre A-S (Conway, Amel, y Gerwien, 2009; Tapia, 2006) y mentoría (Alonso, Castaño, Calles, y Sánchez, 2010), así como una mayor autoestima (McIntyre y Sellnow, 2014; Wilczenski y Coomey, 2007), constructo necesario para un adecuado desarrollo personal, fomentando la reflexión y el pensamiento crítico, así como un papel activo y reflexivo en todo el proceso de toma de decisiones (Puig et al., 2007; Wilczenski y Coomey, 2007), aunque para ello, hubiese sido necesario emplear otros instrumentos para medir dichas variables (p.e., expedientes académicos de los mentores), así como un grupo control con el que contrastar estas medidas y dar mayor rigurosidad al trabajo. Además, comprobar que los resultados logrados por los mentores y la calidad del trabajo realizado durante las sesiones de mentoría, están relacionados con los resultados de impacto de sus respectivos "mentees" (p.e., calificaciones de notas, características socio-ambientales y relaciones personales en el aula).

Respecto a los "mentees" no se ha obtenido el efecto esperado sobre el rendimiento escolar, pero si se realiza un análisis exhaustivo, caso a caso, se aprecian tendencias de mejora en un considerable tanto por ciento de los componentes de la submuestra (55\%), sobre todo en aquellos "mentees" que desarrollaron más de 14 sesiones de mentoría, donde estas tendencias de mejora se ven reflejadas en la áreas instrumentales, como sucede en otros trabajos (Klaw, Fitzgerald, y Rhodes, 2003), ya que la gran mayoría de las actuaciones estaban dirigidas a reforzar las áreas 
curriculares, mejorar las estrategias de aprendizaje y la actitud hacia el estudio (p.e., motivación hacia el estudio).

Asimismo, se perciben ciertos cambios significativos en las características socio-ambientales y relaciones personales en el aula de los "mentees", es decir, se ha observado un leve incremento de la cantidad y calidad de sus interacciones y, por tanto, cierta optimización del clima social, lo cual lleva asociado a un incremento del éxito o rendimiento escolar (Hadre, Crowson, Debacker, y White, 2007) y el bienestar emocional del alumnado (Reddy, Rhodes, y Mulhall, 2003), como consecuencia de los cambios adaptativos que se producen en el comportamiento de los docentes y alumnado, generando un incremento en la motivación, competencias y logros escolares, compromiso escolar, valor a la escuela, y comportamientos adecuados (Reddy et al., 2003).

En definitiva, los resultados refuerzan que el trabajo realizado por los mentores en las sesiones de mentoría ha generado satisfacción y mejoras en los "mentees", fortaleciendo sus habilidades para mejorar sus relaciones personales con el grupo de iguales y docentes, favorecer un mejor clima dentro del aula, y potenciar su desarrollo personal. No obstante, estos resultados se deben interpretar con cautela, teniendo en cuenta que no se han desarrollado el mismo número de sesiones, no presentaban las mismas necesidades, y, por tanto, no han sido objeto de las mismas medidas de intervención. Por ello, sería interesante incluir en próximas ediciones medidas dirigidas a implementar un mayor entrenamiento en habilidades y competencias para atender las necesidades de los "mentees", incrementar el número de sesiones, partir de los intereses de los "mentees", con el propósito de incrementar su motivación, habilidades, competencias, y actitudes, e incrementar el compromiso, coordinación y participación por parte de todos los participantes.

\section{REFERENCIAS}

Alonso, M. A., Castaño, G., Calles, A. M., y Sánchez, S. (2010). Assessment of the Efficacy of a Peer Mentoring Program in a University Setting. The Spanish Journal of Psychology, 13(2), 683-694. doi:http://dx.doi.org/10.1017/S1138741600002353

Arco, J. L. y Fernández, F. D. (2007). Evaluación de programas en educación no formal. En César Torres y José Antonio Pareja (Coord.), La educación no formal y diferenciada. Fundamentos didácticos y organizativos (pp. 667-690). Madrid: Editorial CCS.

Ato, M. (2010). Tipología de diseños cuasiexperimentales. En Mํㅡㄹ Teresa Anguera, Jaume Arnau, Manuel Ato, Rosario Martínez, Juan Pascual y Guillermo Vallejo (Eds.), Métodos de investigación en Psicología (pp. 245-269). Madrid: Síntesis.

Boland, J. (2010). Teaching and learning through civic engagement: Prospects for sustainability in teacher education. Issues in Educational Research, 20(1), 1-20.

Consejo Económico y Social de España (2017). Economía, trabajo y Sociedad. Memoria sobre la situación socioeconómica y laboral. España 2016 (Resumen ejecutivo). Madrid, Consejo Económico y Social.

Conway, J. M., Amel, E. L., y Gerwien, D. P. (2009). Teaching and learning in the social context: A meta-analysis of service learning's effects on academic, personal, social, and citizenship outcomes. Teaching of Psychology, 36(4), 233-245. doi:10.1080/00986280903172969.

Fernández, F.D. (2007). La tutoría entre compañeros en la Universidad (Tesis inédita de doctorado). Universidad de Granada, Granada.

Freire, M. J., Teijeiro, M. M., y País, C. (2013). La adecuación entre las competencias adquiridas por los graduados y las requeridas por los empresarios. Revista de Educación, 362, 13-41. doi:10.4438/1988-592X-RE-2011-362-151

Gismero, E. (2000). Escala de habilidades sociales. Madrid: TEA. 
Hadre, P. L., Crowson, M. H., Debacker, T. K., y White, D. (2007). Predicting the academic motivation of rural high school students. Journal of Experimental Education, 75(4), 247-269. doi:10.3200/JEXE.75.4.247-269

Hervás, M., Fernández, F. D., Arco, J. L., y Miñaca, M. I. (2017). Efectos de un programa de Aprendizaje-Servicio en el alumnado universitario. Electronic Journal of Research in Educational Psychology, 15(41), 126-146. 7. doi:http://dx.doi.org/10.14204/ejrep.41.16049

Instituto Nacional de Evaluación Educativa (2015). Sistema estatal de indicadores de la Educación 2015. Madrid: Ministerio de Educación, Cultura y Deporte.

Jackevicius, C. A., Le, J., Nazer, L., Hess, D., Wang, J., y Law, A. V. (2014). A formal mentorship program for faculty development. American Journal of Pharmaceutical Education, 78(5), 1-10. doi: $10.5688 /$ ajpe785100

Klaw, E. L., Fitzgerald, L. F., y Rhodes, J. E. (2003). Natural mentors in the lives of African-American adolescent mothers: Tracking relationships over time. Journal of Youth and Adolescence, 32(3), 223-232.

Ledesma, R., Macbeth, G., y Cortada, N. (2008). Tamaño del efecto: Revisión teórica y aplicaciones con el sistema estadístico ViSta. Revista Latinoamericana de Psicología, 40(3), 425-439.

Maloney, S. M., y Griffith, K. (2013). Occupational therapy students' development of therapeutic communication skills during a service-learning experience. Occupational Therapy in Mental Health, 29, 10-26. doi:10.1080/0164212X.2013.760288

Maloney, S. M., Myers, C., y Bazyk, J. (2014). The influence of a community-based service-learning experience on the development of occupational therapy students' feelings of civic responsibility. Occupational Therapy in Mental Health, 30(2), 144-161. doi:10.1080/0164212X.2014.910160

Martínez, R. (2010). El método de encuestas por muestreo: Conceptos básicos. En Mํㅡㄹ Teresa Anguera, Jaume Arnau, Manuel Ato, Rosario Martínez, Juan Pascual y Guillermo Vallejo (Eds.), Métodos de investigación en Psicología (pp. 385-43). Madrid: Síntesis.

McIntyre, K. A., y Sellnow, D. D. (2014). A little bit can go a long way: An examination of required service in the basic communication course. Communication Teacher, 28(1), 57-73. doi:10.1080/17404622.2013.843012

Mentor/National Mentoring Partnership. (2005). Cómo construir un programa de mentoría exitoso utilizando los elementos de la práctica efectiva. Alexandria, VA: Mentor/National Mentoring Partnership.

Ministerio de Educación, Cultura y Deporte (2017). Sistema Estatal de Indicadores de Educación (SEIE). Madrid: Subdirección Estatal de Estadística y Estudios e Instituto Nacional de Estadística.

Miñaca, M I. y Hervás, M. (2013). Intervenciones dirigidas a la prevención del fracaso y abandono escolar. Un estudio de revisión. Revista de Educación Comparada, 21, 203-220. doi:http://dx.doi.org/10.5944/reec.21.2013.7620

Moos, R. H., Moos, B. S., y Trickett, E. J. (1995). Escala de Clima Social: Centro Educativo (CES). Madrid: TEA Ediciones.

Pintrich, P., Smith, D., García, T., y McKeachie, W. (1991). A manual for the use of the Motivated Strategies for Learning Questionnaire (MSLQ). Michigan, MI: National Center for Research to Improve Postsecondary Teaching and Learning. University of Michigan.

Puig, J. M., Batlle, R., Bosch, C., y Palos, J. (2007). Aprendizaje-servicio. Educar para la ciudadanía. Barcelona: Octaedro.

Reddy, R., Rhodes, J. E., y Mulhall, P. (2003). The influence of teacher support on student adjust- 
ment in the middle school years: A latent growth curve study. Development and Psychopathology, 15(1), 119-138. doi:http://dx.doi.org/10.1017.S0954579403000075.

Sandford, S., Copps, J., y Yeowart, C. (2007). Lean on me. Mentoring for young people at risk. A guide for donors and funders. London: New Philantropy Capital.

Stanton, T. K. (2014). Practice makes perfect: Classrooms and curricula that generate civic minds and actions. The Journal of General Education, 63(1), 35-46. doi:10.1353/jge.2014.0001

Tapia, M. N. (2006). Aprendizaje y servicio solidario: En el sistema educativo y las organizaciones juveniles. Buenos Aires: Ciudad Nueva.

Vaknin, L. W., y Bresciani, M. J. (2013). Implementing quality service-learning programs in Community Colleges. Community College Journal of Research and Practica, 37(12), 979-989. doi:10.1080/10668926.2010.515515

Vallés, M. S. (2000). Técnicas Cualitativas de Investigación Social. Reflexión Metodológica y Práctica Profesional. Madrid: Síntesis.

Weiler, L. M., Zarich, K. J., Haddock, S. A., Krafchick, J. L., y Zimmerman, T. S. (2014). A comprehensive model of mentor experiences: Perceptions, strategies, and outcomes. Journal of Community Psychology, 42(5), 593-608. doi:10.1002/jcop.21640.

Westover, J. H. (2012). Academic service-learning across disciplines. Models, outcomes and assessment. Champaign, IL: Common Groum Publishing.

Wilczenski, F. L., y Coomey, S. M. (2007). A practical guide to service learning: Strategies for positive development in schools. New York, NY: Springer. 
